\title{
Thermodynamic and kinetic investigation of the influence of external centrifugal field and the heat transfer on a confined neutral gas
}

\author{
Taha Zakaraia Abdel Wahid' ${ }^{1}$ (D) Fatma M. El-Malky²
}

Received: 20 January 2020 / Accepted: 20 March 2020 / Published online: 1 April 2020

(c) Springer Nature Switzerland AG 2020

\begin{abstract}
In the present paper, we offer a new model studying the influence of an external centrifugal field and the heat transfer on a neutral gas confined between two parallel horizontal rigid stationary plane plates. The system of nonlinear differential equations is solved analytically. The temperature and concentration are calculated with a broad range of Knudsen numbers, centrifugal Mach number, and temperatures ratio parameter. We utilized the moment method with suitable boundary conditions. Thermodynamic predictions and internal energy change are examined. Results are applied for laboratory argon gas. Three-dimensional, two-dimensional, and contour plots graphics are drawn, and their manner is discussed.
\end{abstract}

Keywords Kinetic theory - Rarefied gas - Gas centrifuge · Heat transfer - Non-equilibrium distribution functions · BGK model · Exact solutions · Partial differential equations · Moment method · Irreversible non-equilibrium thermodynamics · Internal energy change

Mathematics Subject Classification 76P05 $\cdot 82 \mathrm{~B} 40 \cdot 82 \mathrm{~B} 35 \cdot 82 \mathrm{~B} 23 \cdot 82 \mathrm{~B} 30 \cdot 82 \mathrm{C} 40 \cdot 82 \mathrm{C} 70 \cdot 35 \mathrm{~J} 66$

\section{List of symbols}

C Velocity of the gas particles

$\mathrm{d} \underline{\mathrm{C}}$ Volume element in velocity space, $\mathrm{d} \underline{C}=\mathrm{d} C_{x} \mathrm{~d} C_{y} \mathrm{~d} C_{z}$

$\varphi \quad$ Distribution function

$\varphi_{0} \quad$ Local Maxwellian equilibrium distribution function

$\varphi_{1} \quad$ Distribution function for upward going particles $C_{y}>0$

$\varphi_{2} \quad$ Distribution function for downward going particles $C_{y}<0$

$F_{C} \quad$ Centrifugal force component along $y$-axis direction

J Entropy flux vector

$J_{y} \quad$ Entropy flux component along $y$-axis direction

$K_{\mathrm{B}} \quad$ Boltzmann constant (erg/K)

$K_{\mathrm{n}} \quad$ Knudsen number

$L_{i j} \quad$ Kinetic coefficients

$m$ Particles mass $m_{\mathrm{w}} \quad$ Molecular weight

$n \quad$ Mean density

$n_{\mathrm{s}} \quad$ Concentration at the plate surface

$n_{1} \quad$ Concentration related to $\varphi_{1}$

$n_{2} \quad$ Concentration related to $\varphi_{2}$

$P \quad$ Pressure

$P_{\mathrm{s}} \quad$ Pressure at the plate surface

$P_{y y} \quad$ Pressure deviator

$\Psi_{i} \quad$ The function of the molecular velocity

$R \quad$ Gas constant (erg/deg-mol)

$S \quad$ Entropy per unit mass

$T \quad$ Mean temperature

$T_{s} \quad$ Temperature at the plate surface

$T_{1} \quad$ Temperature related to $\varphi_{1}$

$T_{2} \quad$ Temperature related to $\varphi_{2}$

$u \quad$ Flow (bulk) velocity

$U \quad$ Internal energy of the gas

dU Internal energy changes

$\triangle$ Taha Zakaraia Abdel Wahid, taha_zakaraia@yahoo.com; Taha.Zakaria@science.menofia.edu.eg| ${ }^{1}$ Mathematics and Computer Science Department, Faculty of Science, Menoufia University, Shebeen El-Koom 32511, Egypt. ${ }^{2}$ Faculty of Science, Al-Azhar University for Girls, Nasr City, Cairo 11471, Egypt. 
$\mathrm{d} U_{F} \quad$ Centrifugal force energy change

$\mathrm{d} U_{S}$ Internal energy variation due to a change in entropy

$\mathrm{d} U_{V} \quad$ Internal energy variation due to a change in concentration

$\checkmark \quad$ Gas volume

$V_{\text {Th }} \quad$ Thermal gas velocity

$X_{1} \quad$ Thermodynamic force corresponding to a change in concentration

$X_{2} \quad$ Thermodynamic force corresponding to a change in temperature

y Displacement variable

\section{Greek symbols}

$\eta \quad$ Dimensionless temperature ratio

$\lambda \quad$ Mean free path

$\mu_{s} \quad$ Viscosity coefficient

$\rho \quad$ Gas density

$\sigma \quad$ Entropy production

$\tau \quad$ Relaxation time

$\tau_{x y} \quad$ Shear stress

\section{Superscript}

* Dimensionless variable

\section{Subscripts}

$s \quad$ Boundary value at the palate

$x \quad$ Component along the $x$-axis direction

$y \quad$ Component along the $y$-axis direction

$z \quad$ Component along the $z$-axis direction

\section{Introduction}

Increasing the separation adequacy of a neutral gas influenced by a centrifugal field is one of the vital objectives in uranium gas isotope separation, which is an essential process. The gas isotope separation has various applications in the nuclear operators, electrical power generator, many medical vital applications, and military weapons. Thermal heat transfer plus centrifugal field, affected on a gas confined between rotating cylinders or in its corresponding approximate model, like Pomraning's model, is essential for the gas isotope separation process. In the presence of an external centrifuge field applied in a separating isotope, a countercurrent circulating the gas particles is produced by heating the upper rotor cover and cooling the bottom one. This countercurrent is because the heat transfer makes considerable separation possible in a single centrifuge [1]. Pomraning's model [2-6] acts as if a gas situation between two cylinders can be approximated by a gas situation between two parallel rigid plane plates. In this case, the boundaries move in the opposite direction with equal speeds. The centrifugal force is

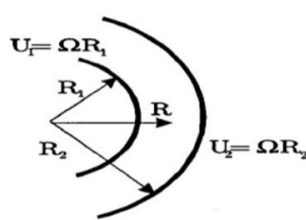

(a)

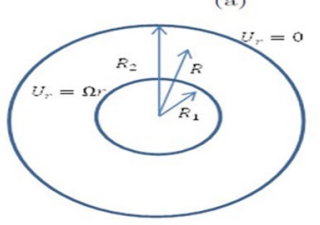

(a)

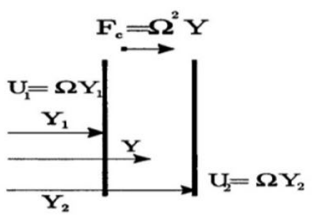

(b)

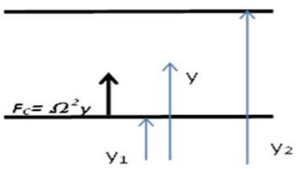

(b)
Fig. 1 Pomraning's strip approximation model illustrated in (b) for the cylindrical geometry illustrated in (a). $F c=\Omega^{2} y$ is the imposed external mass force per unit mass [3]

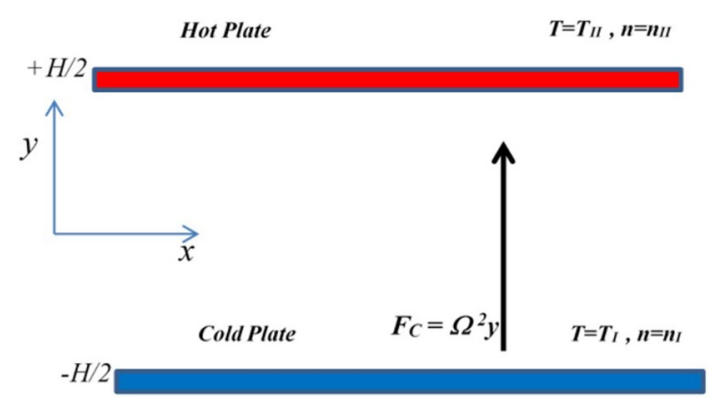

Fig. 2 Geometry of our model of the problem, here $F_{C}=\Omega^{2} y$ is the imposed external mass force per unit mass, $T_{\|}, T_{1}, n_{\|}, n_{1}$ are constants at the surfaces of the two plates as $T_{\|}>T_{1}$

simulated by affecting a mass force perpendicular to the plates and symmetrical to the distance from the uniformity plane. We can say the outward action is the same as a rigid body. Pomraning's geometrical model is illustrated in Fig. 1a, b [3]. Our model is corresponding to a problem as if a gas confined between two cylinders: The outer is stationary, and the inner is rotating with angle velocity $\Omega$ producing a centrifugal force $F_{C}$ directed from the inner to the outer cylinder. So, our model represents an issue of a gas confined between two plane plates suffering from an external centrifugal force perpendicular to the bottom plate directed to the upper one, as shown in Figs. 1 and 2.

The Boltzmann equation $[7,8]$ is suitable for the whole range of Knudsen numbers $\left(K n=\frac{\lambda}{H}\right.$ is defined as the ratio between the mean free path of gas molecules $\lambda$ to the characteristic length $\mathrm{H}$ of the system or representative physical length scale. It is a dimensionless number that examines the gas rarefaction), while the Navier-Stokes equations are valid to give useful results only in the continuum regime, and the gas rarefaction 
is divided into four essential schemes according to the Knudsen number value which are $K n<0.01$ continuum regime, $0.01<K n<0.1$ slip regime, $0.1<K n<10$ transition regime, and finally $K n>10$ free molecular scheme. As the separation processes of a neutral gas influenced by a centrifugal field had many systems in the same problem [3-6] such as rarified and continuum regimes, the Boltzmann kinetic equation should investigate it rather than the Navier-Stokes equations. For larger $K n$, the Navier-Stokes equations are broken down, and the Boltzmann equations or the methods based on the kinetic theory must be applied [9-11]. The large values of the Knudsen numbers are happening in rarefied gases with high magnitude of the mean free path or a minimal amount of the characterized length that occurs in the many micro-electromechanical systems or nanoelectromechanical systems (MEMS or NEMS) devices. That property gives the Boltzmann equation an excellent advantage and various modern applications rather than the Navier-Stokes equations $[12,13]$. Many papers were dealing with Boltzmann kinetic equation and its applications in many critical physical situations such as heat transfer between two parallel plates $[14,15]$, shock waves [16], irreversible thermodynamics [17], sound propagation in a rarefied gases [18], oscillatory flow [19, 20], thermal radiation [21-23], plasma [24], and other various amazing applications [25-27].

In a gas centrifuge, it is necessary to calculate the collision frequency of the gas particles as a function of position within the device. Due to centrifugal force, the gas particles are distributed in a very non-uniform manner between the top plate and the bottom one. Indeed, investigations indicate that while the highdensity region near the top plate can be treated as a continuum regime, the area near the bottom behaves like a rarefied gas regime. Such manner calls for special techniques based upon the Boltzmann kinetic theory description of the gas particle's behavior [1-6]. Pomraning $[2,3]$ has proposed a useful model dealing with the centrifugal field acting on a neutral gas in the plane geometry using a demonstrated force term in the Boltzmann kinetic equation to simulate the centrifugal effect. L. M. De Socio et al. [3] showed that the comparisons display the agreement between the simulations and the experiment is excellent in the continuum regime, while it gives a very good one in the transitional system. A full review of previous works dealing with the applications of the kinetic theory related to this problem can also be found in E.A. Johnson and L. M. De Socio's papers [3-5].

We aim to investigate the effectiveness of an external centrifugal force and heat transfer on the manner of the particles' non-equilibrium distribution function of a neutral gas confined between two parallel horizontal rigid stationary plates. These investigations are done in a wide range of Knudsen numbers, more specific in the transition, the hydrodynamic and the rarefied gas regimes, and a broad range of centrifugal Mach. Our calculations have been done with a wide range of temperatures ratio parameters between the two parallel plates. For this propose, an exact analytical solution of the BGK (Bhatnagar-Gross-Krook) kinetic model has introduced with the two-sided non-equilibrium distribution Maxwellian functions. In a frame co-moving with the fluid $[9,21-23]$, the system consisting of four nonlinear partial differential equations is solved analytically. The moment method [28-30] with suitable boundary conditions is applied. The manner of the macroscopic properties of the neutral gas such as temperature and concentration is investigated. We calculated the non-equilibrium distribution functions to discuss the non-equilibrium thermodynamic properties of the whole system (neutral gas particles + the two plates). The internal energy change is examined. The results applied for laboratory argon gas. Three-dimensional, two-dimensional, and contour plot graphics that illustrate the investigation are drawn to predict their manner. The results are discussed, and agreements with related studies are shown.

\section{Basic equations and analytical solution procedures}

We have treated the neutral gas between two infinite horizontal parallel plates located at $y= \pm H / 2$, as shown in Fig. 2. The two plates are kept at two constants, but different temperatures as $T_{1}$ for the bottom plate and $T_{\|}$ for the top plate with $T_{1}=\eta T_{\|}, 0<\eta \leq 1$, where $\eta$ is the temperature ratio parameter. In a frame co-moving with the fluid [21-23], the manner of the neutral gas is studied under postulating that the heat exchange will be due to only the temperature difference between the particles and the heated plates, taking the form of full-energy accommodation [1]. We have treated the case in which there exists a centrifugal force $\left(F_{c}=m \Omega^{2} y\right)$ perpendicular to the parallel plates directed from the bottom plate to the top one, as illustrated in Fig. 2. For the steady motion, a centrifugal force $F_{C}$ can be expressed in terms of the Boltzmann equation [28-30] in the BGK model as:

$C_{y} \frac{\partial \varphi}{\partial y}+\frac{F_{y}}{m} \frac{\partial \varphi}{\partial C_{y}}=\frac{\left(\varphi_{0}-\varphi\right)}{\tau}$,

as

SN Applied Sciences A SPRINGER NATURE journa 
$\varphi_{0}=\frac{n(y)}{(2 \pi R T(y))^{\frac{3}{2}}} \exp \left[\frac{-C^{2}}{(2 R T(y))}\right]: C^{2}=C_{x}^{2}+C_{y}^{2}+C_{z}^{2}$.

Maxwell converted the Maxwell-Boltzmann equation into an integral equation of transfer for any quantity $\Psi_{i}$ that is a function only of the molecular velocity. The distribution function $\varphi$ utilized here should be considered as a suitable weighting function, making use of the Liu-Lees model of the two-stream Maxwellian distribution function in the form [31, 32]:

$\varphi=\left\{\begin{array}{l}\varphi_{1}=\frac{n_{1}(y)}{\left(2 \pi R T_{1}(y)\right)^{\frac{3}{2}}} \exp \left[\frac{-C^{2}}{\left(2 R T_{1}(y)\right)}\right], \text { For } \quad C_{y}>0 \\ \varphi_{2}=\frac{n_{2}(y)}{\left(2 \pi R T_{2}(y)\right)^{\frac{3}{2}}} \exp \left[\frac{-C^{2}}{\left(2 R T_{2}(y)\right)}\right], \text { For } \quad C_{y}<0 \quad \downarrow\end{array}\right.$

Maxwell's moment equations are derived by multiplying the BGK equation by a function of a velocity $\Psi_{i}(C)$ and then integrating over the velocity space. Multiplying Eq. (2) by some functions of velocity $\Psi_{i}=\Psi_{i}(C)$ and integrating concerning $C$, taking into consideration the two-sided distribution function [31, 32], the obtained transfer equation can be written as: which represents the number density.

$u(y)=\frac{1}{n} \int C_{y} \varphi\left(y, C_{x}, C_{y}, C_{z}\right) \mathrm{d} \underline{C}=\frac{\left(n_{1} \sqrt{T_{1}}-n_{2} \sqrt{T_{2}}\right)}{\left(n_{1}+n_{2}\right)}$,

which represents the hydrodynamic (bulk) velocity.

$T(y)=\frac{1}{3 n} \quad \int C^{2} \varphi\left(y, C_{x}, C_{y}, C_{z}\right) \mathrm{d} \underline{C}=\left(\frac{n_{1} T_{1}+n_{2} T_{2}}{n_{1}+n_{2}}\right)$,

which represents the temperature.

$P_{y y}(y)=\int C_{y}^{2} \quad \varphi\left(y, C_{x}, C_{y}, C_{z}\right) \mathrm{d} \underline{C}=\frac{1}{2}\left(n_{1} T_{1}+n_{2} T_{2}\right)$,

which represents the static pressure.

$q_{y}(y)=\int C_{y} C^{2} \varphi\left(y, C_{y}\right) \mathrm{d} \underline{C}=\left(n_{1} T_{1}^{\frac{3}{2}}-n_{2} T_{2}^{\frac{3}{2}}\right)$,

which represents the heat flux component.

In Eq. (4), there are four uncharted functions $T_{1}(y), T_{2}(y), n_{1}(y)$ and $n_{2}(y)$ wanted to be obtained. For this proposal, we need four equations to be solved. Two

$$
\begin{aligned}
\frac{\partial}{\partial y}\left[\int_{-\infty}^{\infty} \int_{-\infty}^{0} \int_{-\infty}^{\infty} \Psi_{i} C_{y} \varphi_{2} \mathrm{~d} \underline{C}+\int_{-\infty}^{\infty} \int_{0}^{\infty} \int_{-\infty}^{\infty} \Psi_{i} C_{y} \varphi_{1} \mathrm{~d} \underline{C}\right]-\frac{F_{y}}{m}\left(\int_{-\infty}^{\infty} \int_{-\infty}^{0} \int_{-\infty}^{\infty} \varphi_{2} \frac{\mathrm{d} \Psi_{i}}{\mathrm{~d} C_{y}} \mathrm{~d} \underline{C}+\int_{-\infty}^{\infty} \int_{0}^{\infty} \int_{-\infty}^{\infty} \varphi_{1} \frac{\mathrm{d} \Psi_{i}}{\mathrm{~d} C_{y}} \underline{\mathrm{d} C}\right) \\
\quad=\frac{1}{\tau}\left[\int_{-\infty}^{\infty} \int_{-\infty}^{\infty} \int_{-\infty}^{\infty} \Psi_{i} \varphi_{0} \mathrm{~d} \underline{C}-\left(\int_{-\infty}^{\infty} \int_{-\infty}^{0} \int_{-\infty}^{\infty} \varphi_{2}+\int_{-\infty}^{\infty} \int_{0}^{\infty} \int_{-\infty}^{\infty} \varphi_{1}\right) \Psi_{i} \mathrm{~d} \underline{C}\right], \text { where } \mathrm{d} \underline{C}=\mathrm{d} C_{x} \mathrm{~d} C_{y} \mathrm{~d} C_{z}
\end{aligned}
$$

Dimensionless forms of the variables can be obtained by:

$$
\begin{gathered}
y=y^{*}\left(\frac{H}{2}\right), \vec{C}=\vec{C}^{*} \sqrt{2 R T_{l}}, \varphi_{i}=\frac{\varphi_{i}^{*}\left(2 \pi R T_{l}\right)^{\frac{3}{2}}}{n_{l}}, \quad i=0,1,2, T_{1}=T_{1}^{*} T_{l}, \\
n_{1}=n_{1}^{*} n_{l}, T_{2}=T_{2}^{*} T_{l}, n_{2}=n_{2}^{*} n_{l}, K n=\frac{\sqrt{8 R T_{l}} / \nu_{l}}{H} \text { and } \mathrm{d} U=\mathrm{d} U^{*}\left(K_{B} T_{l}\right) .
\end{gathered}
$$
$\Psi_{i}=C$ and $\frac{1}{2} C_{y} C^{2}$ and substituting Eq. (3) into Eq. (4)

When the formulas $\varphi_{0}, \varphi_{1}$ and $\varphi_{2}$ are presented, macroscopic quantities such as density, velocity, and temperature can be calculated from the integrals of the distribution functions $\varphi$ [33], where:

$n(y)=\int \varphi\left(y, C_{x}, C_{y}, C_{z}\right) d \underline{C}=\frac{\left(n_{1}(y)+n_{2}(y)\right)}{2}$, taking into consideration Eq. (5). Dropping the stars, we acquire the energy and the heat flux balance law equations, respectively:

$$
\frac{\mathrm{d}}{\mathrm{d} y}\left[\left(n_{1} T_{1}^{\frac{3}{2}}-n_{2} T_{2}^{\frac{3}{2}}\right)\right]-\left(M^{2} y\right)\left(n_{1} T_{1}^{\frac{1}{2}}-n_{2} T_{2}^{\frac{1}{2}}\right)=0,
$$




$$
\frac{5}{4} \frac{\mathrm{d}}{\mathrm{d} y}\left(n_{1} T_{1}^{2}+n_{2} T_{2}^{2}\right)-\frac{5}{6}\left(M^{2} y\right)\left(n_{1} T_{1}+n_{2} T_{2}\right)=\frac{e^{-A\left(1-y^{2}\right)}}{K_{n}} \quad\left(n_{1} T_{1}^{\frac{3}{2}}-n_{2} T_{2}^{\frac{3}{2}}\right),
$$

As the collision frequency $v$ in the presence of a centrifugal field had the expression [34-36]:

$v=v_{l} e^{-A\left(1-y^{2}\right)}$, where $v_{l}$ is the collision frequency at the bottom plate, and $A=M^{2} m_{w}$ as $M=\frac{\Omega H}{\sqrt{2 R T}}$ is the centrifugal Mach number and $m_{w}$ is the molecular weight of the neutral gas. The needed equations are accomplished by the equation of state, where:

$P=n T=$ const,

with the condition that we shall study this problem in a coordinate system of phase space as the bulk velocity $u$ is located at the origin $[9,21,22]$.

Then, using Eq. (7) we acquire:

$\left(n_{1} \sqrt{T_{1}}-n_{2} \sqrt{T_{2}}\right)=0$

Solving Eqs. (11-14) to get $T_{1}, T_{2}, n_{1}$ and $n_{2}$ values. From Eq. (14), we obtain:

$n_{2} \sqrt{T_{2}}=n_{1} \sqrt{T_{1}}$.

Substituting Eq. (12) into Eq. (16) yields:

$\frac{\mathrm{d}}{\mathrm{d} y}\left(n_{1} T_{1}^{\frac{3}{2}}-n_{2} T_{2}^{\frac{3}{2}}\right)=\frac{\mathrm{d}}{\mathrm{d} y}\left(n_{2} \sqrt{T_{2}}\left(T_{1}-T_{2}\right)\right)=0$.

Integrating Eq. (17) to acquire, after factorization, that

$$
\left(n_{2} \sqrt{T_{2}}\left(\sqrt{T}_{1}-\sqrt{T}_{2}\right)\left(\sqrt{T}_{1}+\sqrt{T}_{2}\right)\right)=\vartheta_{1} \vartheta_{2}=C_{2}
$$

as

$$
\vartheta_{1}=\left(n_{2} \sqrt{T_{2}}\left(\sqrt{T}_{1}+\sqrt{T}_{2}\right)\right), \vartheta_{2}=\left(\sqrt{T}_{1}-\sqrt{T_{2}}\right),
$$

where $C_{2}$ is the integration constant, depends on the boundary conditions, which comes from the postulating that the pressure is a uniform. Then, $P_{y y}=\frac{n_{2}}{2} \sqrt{T_{2}}\left(\sqrt{T}_{1}+\sqrt{T}_{2}\right)=\frac{\vartheta_{1}}{2}$, and it is independent on $y$. That implies that $\vartheta_{2}$ is constant as well. For sampling our calculation, we define a function $\zeta(y)$ in the form:

$\zeta(y)=n_{2} \sqrt{T_{2}}=n_{1} \sqrt{T_{1}}$.

From Eqs. (18) and (19), we can acquire:

$$
\begin{aligned}
& T_{1}=\frac{\left(\vartheta_{1}+\vartheta_{2} \zeta\right)^{2}}{4 \zeta^{2}}, T_{2}=\frac{\left(\vartheta_{1}-\vartheta_{2} \zeta\right)^{2}}{4 \zeta^{2}}, \\
& n_{1}=\frac{2 \zeta^{2}}{\left(\vartheta_{1}+\vartheta_{2} \zeta\right)} \text { and } n_{2}=\frac{2 \zeta^{2}}{\left(\vartheta_{1}-\vartheta_{2} \zeta\right)} .
\end{aligned}
$$

Integrating Eq. (12) w. r. t. y and taking into account of Eq. (17), we acquire,

$$
\begin{gathered}
\frac{5}{4}\left(n_{1} T_{1}^{2}+n_{2} T_{2}^{2}\right)-\frac{5}{12}\left(M^{2} y^{2}\right)\left(n_{1} T_{1}+n_{2} T_{2}\right) \\
=\frac{\vartheta_{1} \vartheta_{2}}{K_{n}} \frac{\sqrt{\pi} e^{-A} E r f i[\sqrt{A y}]}{2 \sqrt{A}}+\vartheta_{3}
\end{gathered}
$$

where Erfi[y] gives the imaginary error function $\frac{\operatorname{erf}[i y]}{i}$, in which erf[z] $=\frac{2}{\sqrt{\pi}} \int_{0}^{z} e^{-t^{2}} \mathrm{~d} t$, and $\vartheta_{3}$ is the integration constant, which depends on the boundary conditions. Substituting Eq. (20) into Eq. (21) yields the algebraic equation:

$$
\begin{aligned}
\frac{3}{4} & M^{2} y^{2}\left(\frac{1}{2}\left(\vartheta_{1}-\zeta \vartheta_{2}\right)+\frac{1}{2}\left(\vartheta_{1}+\zeta \vartheta_{2}\right)\right) \\
+ & \frac{5}{4}\left(\frac{\left(\vartheta_{1}-\zeta \vartheta_{2}\right)^{3}}{8 \zeta^{2}}+\frac{\left(\vartheta_{1}+\zeta \vartheta_{2}\right)^{3}}{8 \zeta^{2}}\right) \\
& -\vartheta_{3}+\vartheta_{1} \vartheta_{2} v_{A} \text { Erf }\left[m_{w} M y\right]=0
\end{aligned}
$$

Solving Eq. (22), we acquire the two roots for $\zeta$, as:

$$
\begin{aligned}
& \zeta=\frac{-\sqrt{15} A^{1 / 4} \sqrt{\mathrm{Kn}} \vartheta_{1}^{3 / 2}}{\sqrt{20 \sqrt{A} \mathrm{Kn} M^{2} y^{2} \vartheta_{1}-45 \sqrt{A} \mathrm{Kn} \vartheta_{1} \vartheta_{2}^{2}+48 \sqrt{A} \mathrm{Kn} \vartheta_{3}+48 \mathrm{e}^{A\left(-1+y^{2}\right)} \vartheta_{1} \vartheta_{2} \text { DawsonF }[\sqrt{A y}]}} \\
& \zeta=\frac{+\sqrt{15} A^{1 / 4} \sqrt{\mathrm{Kn}} \vartheta_{1}^{3 / 2}}{\sqrt{20 \sqrt{A} K n M^{2} y^{2} \vartheta_{1}-45 \sqrt{A} K n \vartheta_{1} \vartheta_{2}^{2}+48 \sqrt{A K n} \vartheta_{3}+48 \mathrm{e}^{A\left(-1+y^{2}\right)} \vartheta_{1} \vartheta_{2} \text { DawsonF }[\sqrt{A} y]}}
\end{aligned}
$$


Here, the function DawsonF $[y]$ is the Dawson integral $F(y)$, where [37]

$F(x)=e^{-x^{2}} \int_{0}^{x} e^{y^{2}} \mathrm{~d} y=0.5 \sqrt{\pi} e^{-x^{2}} \operatorname{erfi}(x)$.

We keep the root value that gives positive signs for both temperature and concentration.

The constants values for $\vartheta_{1}, \vartheta_{2}$, and $\vartheta_{3}$ can be calculated using the boundary conditions that have the nondimensional forms:

$\frac{\left(n_{1}(-1)+n_{2}(-1)\right)}{2}=1$,

$\left(\frac{n_{1}(-1) T_{1}(-1)+n_{2}(-1) T_{2}(-1)}{n_{1}(-1)+n_{2}(-1)}\right)=1$,

$n_{1}(-1) \sqrt{T_{1}(-1)}-n_{2}(-1) \sqrt{T_{2}(-1)}=0$

$T_{1}(-1)=\eta T_{2}(-1), 0<\eta \leq 1$

Solving the algebraic system of Eqs. (24-27), we acquire

$\left\{\begin{array}{l}n_{1}(-1)=\frac{2 \sqrt{\eta}}{1+\sqrt{\eta}}, \quad n_{2}(-1)=\frac{2}{1+\sqrt{\eta}}, \\ T_{1}(-1)=\frac{1+\sqrt{\eta}}{\eta+\sqrt{\eta}}, T_{2}(-1)=\frac{\eta(1+\sqrt{\eta})}{\eta+\sqrt{\eta}}\end{array}\right.$.

Substituting Eq. (28) into Eq. (18), we get

$\vartheta_{1}=2, \vartheta_{2}=\eta^{-0.25}-\eta^{0.25}$.

Substituting Eqs. $(28,29)$ into Eq. $(21)$, we obtain

$\vartheta_{3}=\frac{5\left(3-\left(3+M^{2}\right) \sqrt{\eta}+3 \eta\right)}{6 \sqrt{\eta}}+\frac{\vartheta_{1} \vartheta_{2} \text { Dawson } F[\sqrt{A}]}{\sqrt{A} K n}$

The obtained variables in (20) enable us to calculate the distribution functions $\varphi$, which help us to investigate their manner, which is not available by using the Navier-Stokes equations system. This study of the distribution functions will be the starting point to investigate the irreversible thermodynamic properties of the system.

\section{The steady-state non-equilibrium thermodynamic characteristics of the system}

The issues of the thermodynamics of irreversible processes persist an aroused great interest [38]. We should consider that the steady-state non-equilibrium thermodynamic characteristics of the system are investigated under the influence of an external centrifugal force. Entropy per unit mass $S$ is calculated as:

$S=-\int f \log f \mathrm{~d} C=\frac{\pi^{\frac{3}{2}}}{8}\left(n_{1}\left(3-4 \ln \left(\frac{n_{1}}{T_{1}^{\frac{3}{2}}}\right)\right)+n_{2}\left(3-4 \ln \left(\frac{n_{2}}{T_{2}^{\frac{3}{2}}}\right)\right)\right)$,

The $y$-component of entropy flux is

$$
\begin{aligned}
J_{y}=\int C_{y} f \log f \mathrm{~d} \underline{C}= & \frac{\pi}{2}\left(n_{1} \sqrt{T_{1}}\left[1-\ln \left(\frac{n_{1}}{T_{1}^{\frac{3}{2}}}\right)\right]\right. \\
& \left.-n_{2} \sqrt{T_{2}}\left[1-\ln \left(\frac{n_{2}}{T_{2}^{\frac{3}{2}}}\right)\right]\right) .
\end{aligned}
$$

Entropy production in the steady state is expressed as:

$\sigma_{S}=\underline{\nabla} \cdot \underline{J}$

The thermodynamic forces, harmonious with the variation in concentration, temperature and centrifugal force, can be estimated by [39]:

$X_{1}=\frac{H}{n} \frac{\partial n}{\partial y}, X_{2}=\frac{H}{T} \frac{\partial T}{\partial y}, X_{3}=F_{C}$

respectively. Here, $F_{C}=M^{2} y$ is the centrifugal force.

Kinetic coefficients $L_{i j}$ are obtained by the relationship between the entropy production with the thermodynamic forces that has the form [38]:

$\sigma_{S}=\sum_{i} \sum_{j} L_{i j} X_{i} X_{j}=\left(\begin{array}{lll}X_{1} & X_{2} & X_{3}\end{array}\right)\left(\begin{array}{lll}L_{11} & L_{12} & L_{13} \\ L_{21} & L_{22} & L_{23} \\ L_{31} & L_{32} & L_{33}\end{array}\right)\left(\begin{array}{l}X_{1} \\ X_{2} \\ X_{3}\end{array}\right)$.

The Gibb's formula for the variation of the internal energy applied to the system (neutral gas + heated plate) is $\mathrm{d} U=\mathrm{d} U_{S}+\mathrm{d} U_{V}+\mathrm{d} U_{F}$, as the internal energy variation is due to the modification of the temperature, concentration, and centrifugal force variables. Here, $\mathrm{d} U_{S}=T \mathrm{~d} s, \mathrm{~d} U_{V}=-p \mathrm{~d} V, \mathrm{~d} U_{F}=-M^{2} y \mathrm{~d} y$ ， where the pressure and the change in the volume are $P=n T, \mathrm{~d} V=(-\mathrm{d} n) / n^{2}$. 

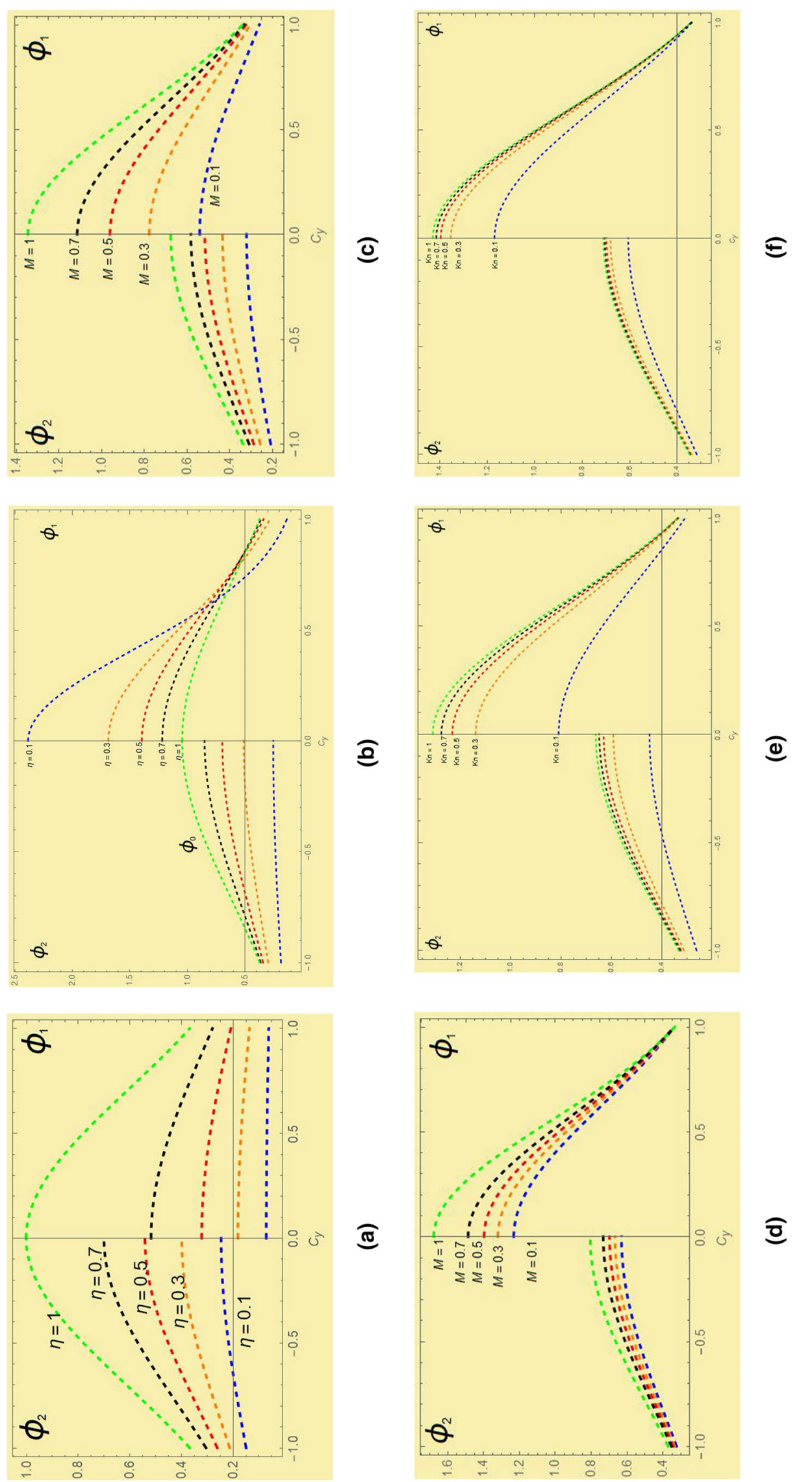

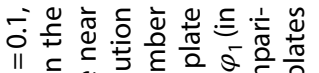

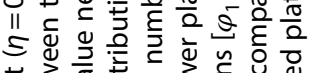

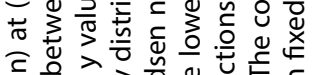

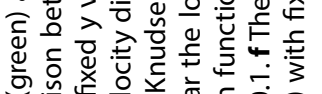

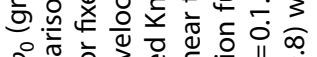

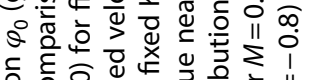

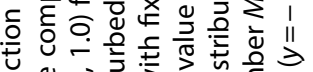

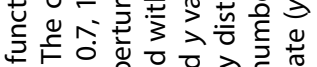

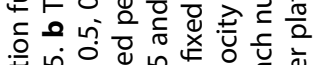

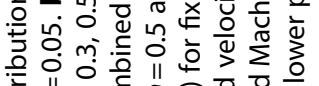

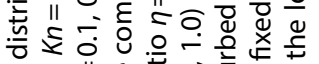

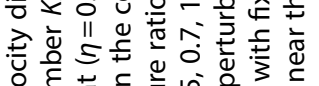

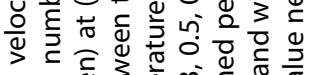

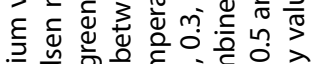

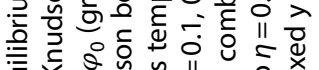

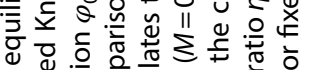
인

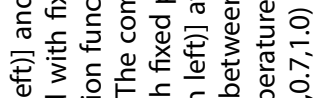

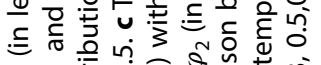
N-

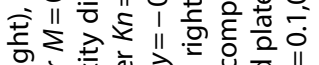

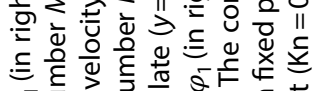

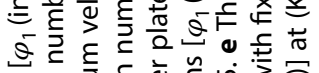

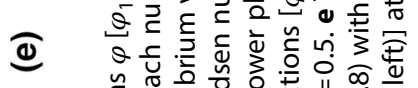

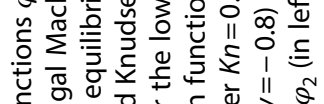

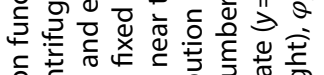

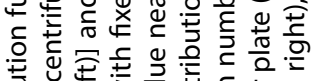

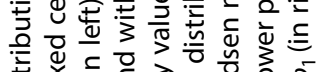

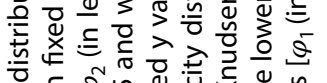

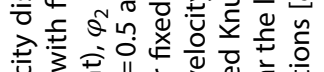
ग

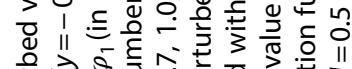

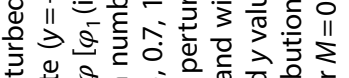

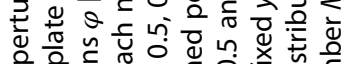

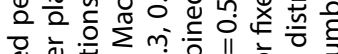

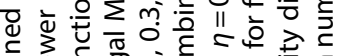

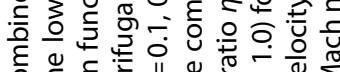

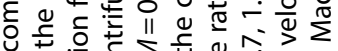

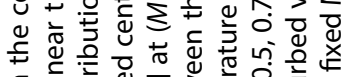

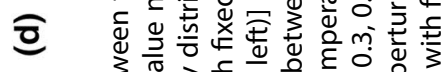

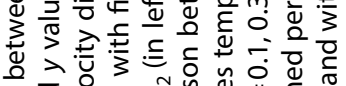
o

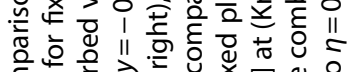

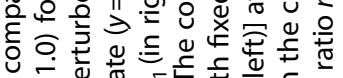

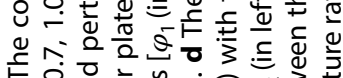
ह m

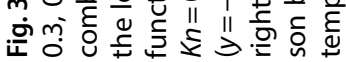




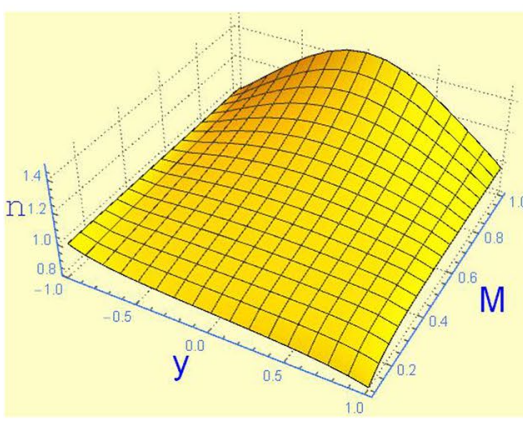

(a)

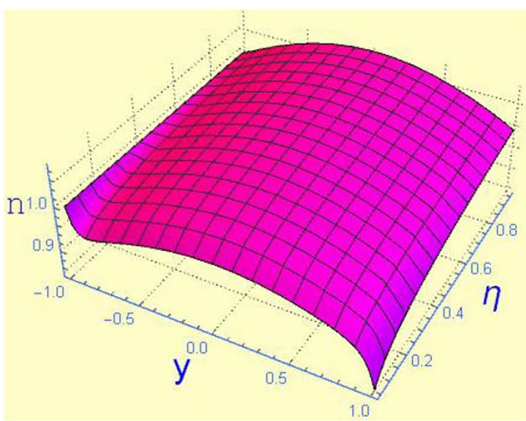

(d)

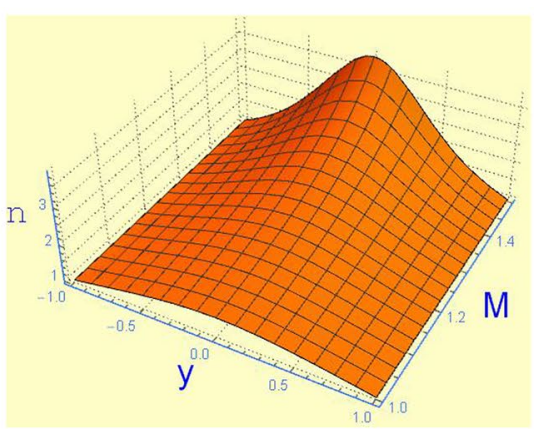

(b)

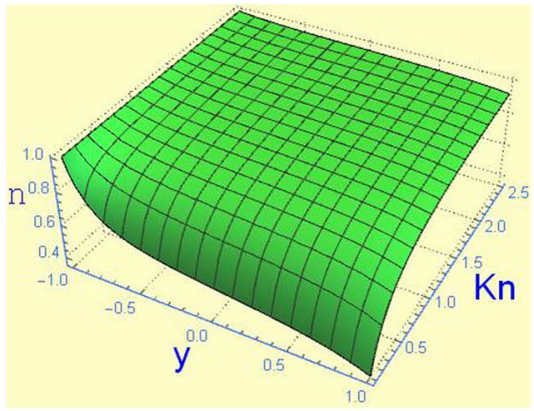

(e)

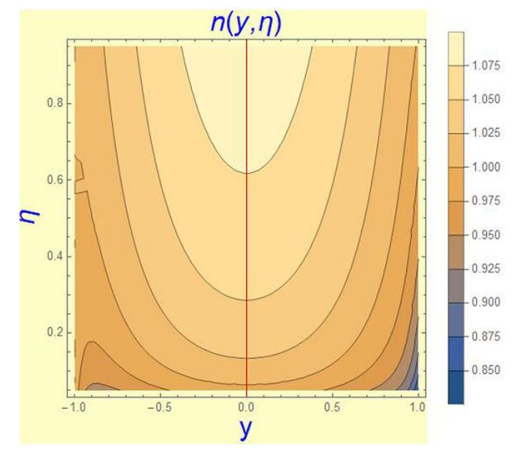

(c)

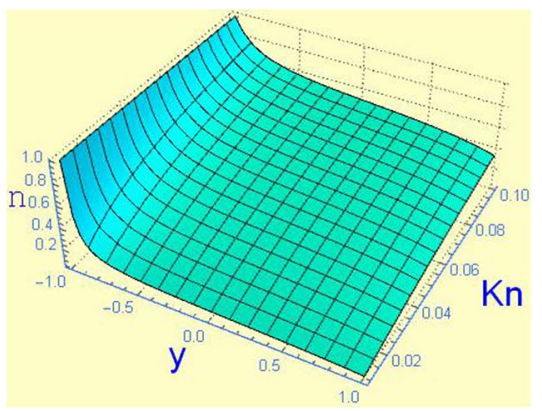

(f)
Fig. 4 a Concentration $n$ versus $y$ and $M$ for a weak and moderated external centrifugal fields $K n=0.75, \eta=0.5$. $\mathbf{b}$ Concentration $n$ versus $y$ and $M$ for a strong external centrifugal field for $K n=0.75, \eta=0.5$. c Contour plot of $n$ versus $y$ and $\eta$ for $K n=0.75, M=0.5$. d Concentra- tion $n$ versus $y$ and $\eta$ for $K n=0.75, M=0.5$. e Concentration $n$ versus $y$ and $K n$ in the transition regime for $M=0.1, \eta=0.5$. $\mathbf{f}$ Concentration $n$ versus $y$ and $K n$ in the slip regime for $M=0.1, \eta=0.5$

\section{Calculation results and discussion concluding remarks}

In a frame co-moving with the gas, the neutral gas manner under the influence of an external centrifugal force and the heat transfer in the steady state is investigated for the system (neutral gas + the heated plates). The external centrifugal force is introduced in the force term of the Boltzmann equation taking that the collision frequency $v$ is a variable dependent on $y$. All calculations are applied for laboratory argon gas with:

- A wide range of Knudsen number $K n$ that is the transition, the hydrodynamic, and the rarefied gas regimes.

- A broad range of centrifugal Mach numbers $M$, which is the strong, the moderated, and the weak fields.

- A full variety of temperatures ratio parameter $\eta$ between the two parallel rigid plates is considered.
Our calculations did not contain any approximations or restrictions on the values of the main controlled parameters (the centrifugal Mach number $M$, the Knudsen number $K n$, and the temperature ratio parameter $\eta)$. Thus, we had a considerable amount of figures corresponding to the broad range of the three controlled parameters. So, when we analyzed the manner of all variables, we will shed light upon some essential public remarks to reduce the discussion.

Figures are performed with some constant values of the non-dimensional parameters that we will illustrate its physical meaning as follows:

- The fixed amount of Knudsen number $K n=0.75$, which represents the transition regime, so the neutral gas is rarefied, is considered in Figs. (A, $B, C, D, 6,7,8,9,10$, $11,12,13$, and 14).

- The fixed amount of Knudsen number $K n=0.05$, which represents the slip regime, so the neutral gas is weakly rarefied, is considered in Fig. 3a-c. 


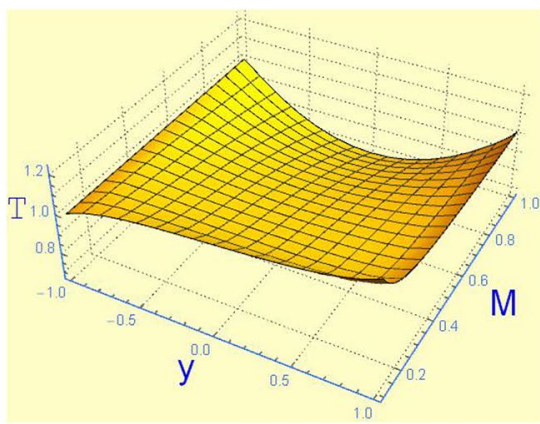

(a)

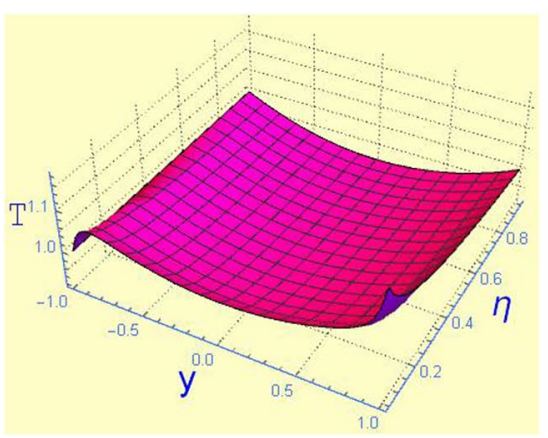

(d)

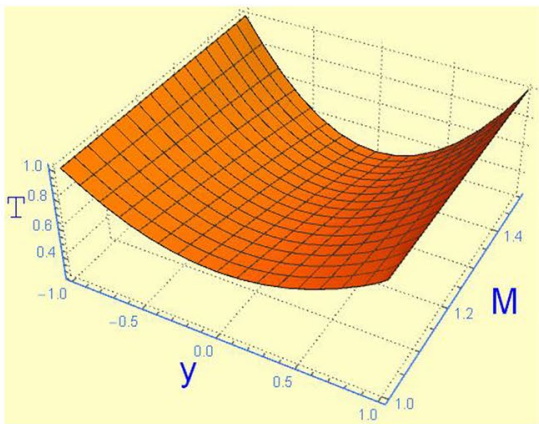

(b)

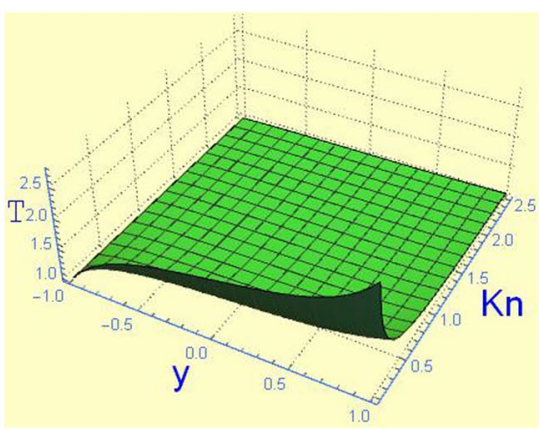

(e)

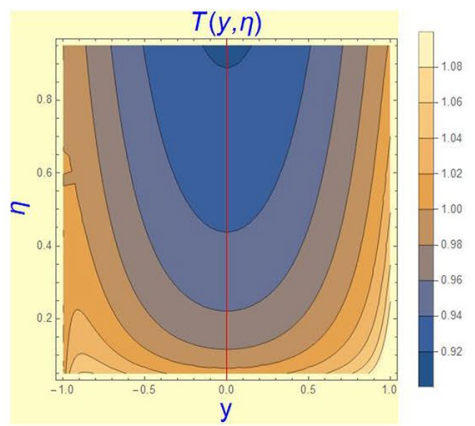

(c)

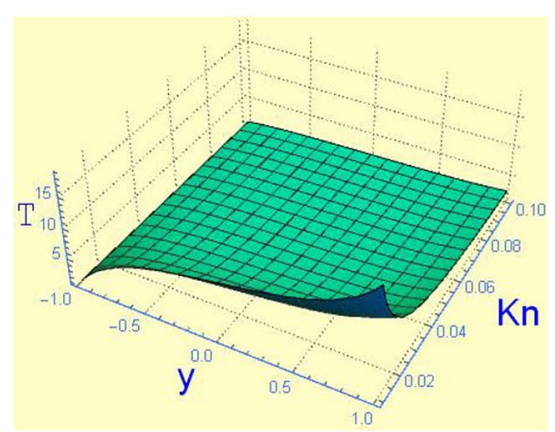

(f)
Fig. 5 a Temperature $T$ versus $y$ and $M$ for a weak and moderated external centrifugal fields $K n=0.75, \eta=0.5$. $\mathbf{b}$ Temperature $T$ versus $y$ and $M$ for a strong external centrifugal field for $K n=0.75, \eta=0.5$. c Contour plot of $T$ ver- sus $y$ and $\eta$ for $K n=0.75, M=0.5$. $\mathbf{d}$ Temperature $T$ versus $y$ and $\eta$ for $K n=0.75$, $M=0.5$. e Temperature $T$ versus $y$ and $K n$ in the transition regime for $M=0.1$, $\eta=0.5$.f Temperature $T$ versus $y$ and $K n$ in the slip regime for $M=0.1, \eta=0.5$
- The fixed value of $\eta=0.5$, which means that the temperature value of the upper plate is two times the temperature value of the lower plate, is considered in Fig. 4a, b, e, f.

- The fixed value of $M=0.5$, which represents the moderate intensity of the external centrifugal force, is considered in Figs. (C, D, 6, 7, 8, 9, 10, 11, 12, 13, and 14).

- The fixed value of $M=0.1$, which represents the weak intensity of the external centrifugal force, is considered in Figs. 3a, 3, 4, 5e, 3, 4, and 5 f.

Figure 3a-f sheds light upon the comparison between the combined perturbed velocity distribution functions and the equilibrium distribution functions in a board range of temperature ratio parameter corresponding various values of Knudsen number and centrifugal Mach number, as shown in Fig. 3a, b. We clarified the manner of the combined perturbed velocity distribution functions and the equilibrium distribution functions in a board range of centrifugal Mach number corresponding various values of Knudsen number and temperature ratio parameter, as shown in Fig. 3c, d.

We illuminate the behavior of the combined perturbed velocity distribution functions and the equilibrium distribution functions in a board range of Knudsen number corresponding various values of centrifugal Mach number and temperature ratio parameter, as shown in Fig. $3 e$, $f$; the decrement in $\varphi_{2}$ and the increment in $\varphi_{1}$ of the distribution functions $\varphi$ are due to the non-equilibrium state according to the effect of the external centrifugal force and the heat transfer between the two rigid plates. That is interpreted as the departure from the equilibrium state, where the gas particles having temperature $T_{1}$ and density $n_{1}$ near the bottom plate after affecting by an external centrifugal force are replaced by a countercurrent of gas particles having temperature $T_{2}$ and density $n_{2}$ near the outer plate after affecting by the heat transfer from the top plate (the hotter one). This manner agrees with Le Chatelier's principle. Figure 3a, b shows, as expected, a coincidence between $\varphi_{0}$ and $\varphi$ at $\eta=1$. That is because, at equilibrium, 


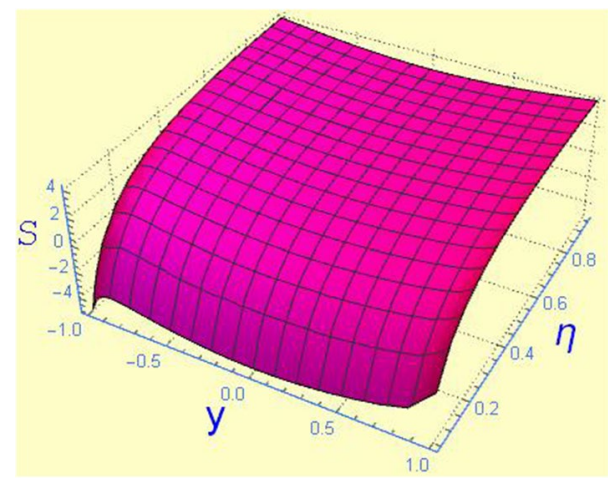

Fig. 6 Entropy $S$ versus $y$ and $\eta$ for $K n=0.75, M=0.5$

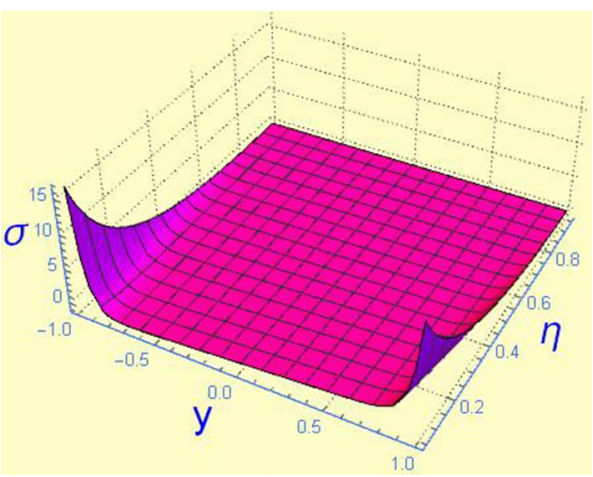

Fig. 7 Entropy production $\sigma$ versus $y$ and $\eta$ for $K n=0.75, M=0.5$

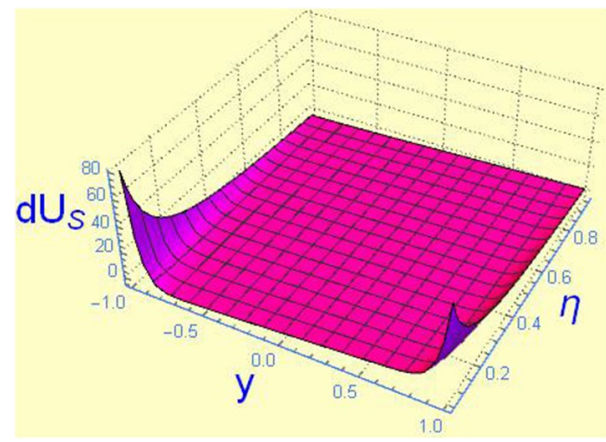

Fig. $8 \mathrm{~d} U_{S} n$ versus $y$ and $\eta$ for $K n=0.75, M=0.5$

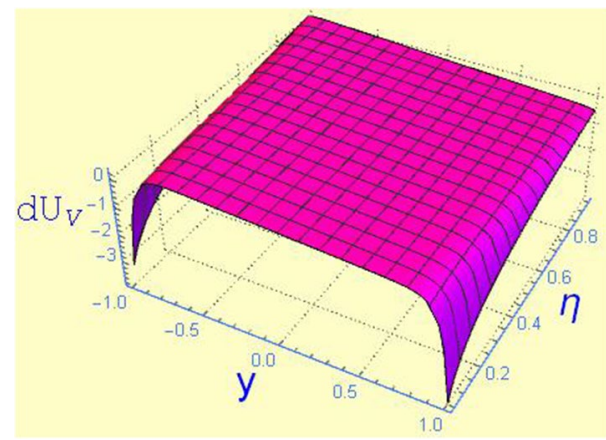

Fig. $9 \mathrm{~d} U_{V}$ versus $y$ and $\eta$ for $K n=0.75, M=0.5$

\section{SN Applied Sciences}

a SPRINGER NATURE journal

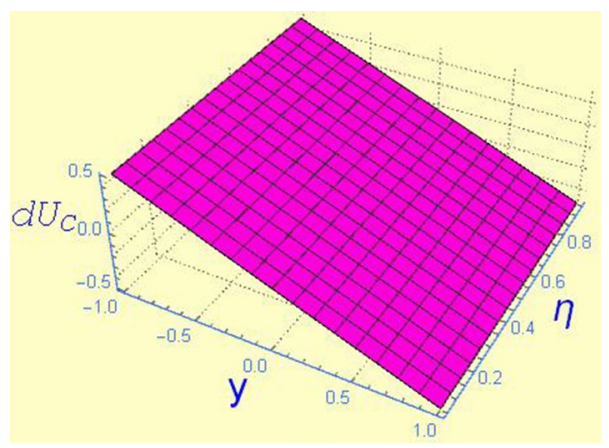

Fig. $10 \mathrm{~d} U_{C}$ versus $y$ and $\eta$ for $K n=0.75, M=0.5$

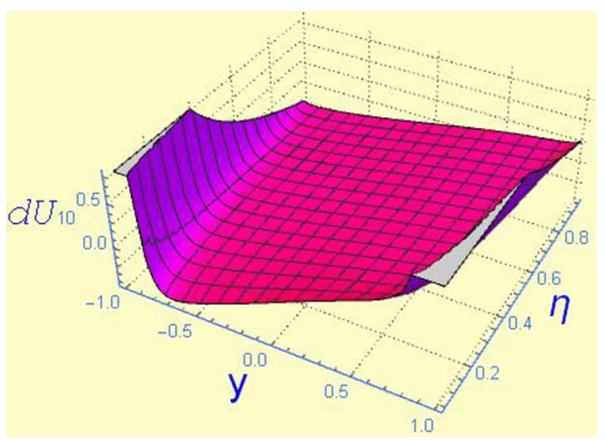

Fig. $11 \mathrm{~d} U$ versus $y$ and $\eta$ for $K n=0.75, M=0.5$

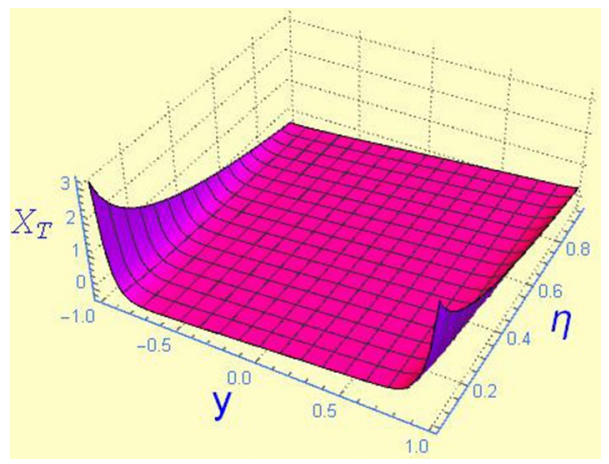

Fig. 12 Thermodynamic force $X_{T}$ versus $y$ and $\eta$ for $K n=0.75, M=0.5$

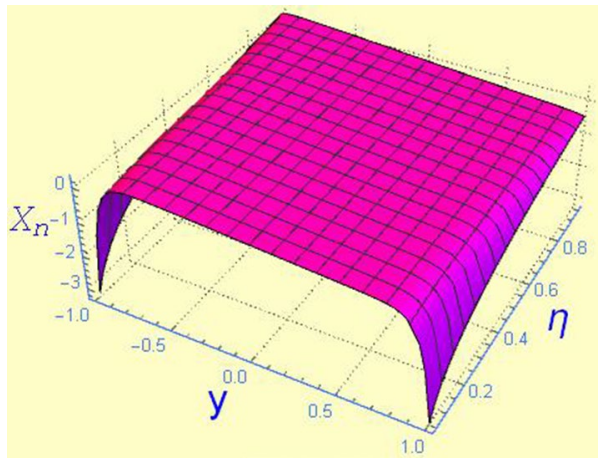

Fig. 13 Thermodynamic force $X_{n}$ versus $y$ and $\eta$ for $K n=0.75, M=0.5$ 


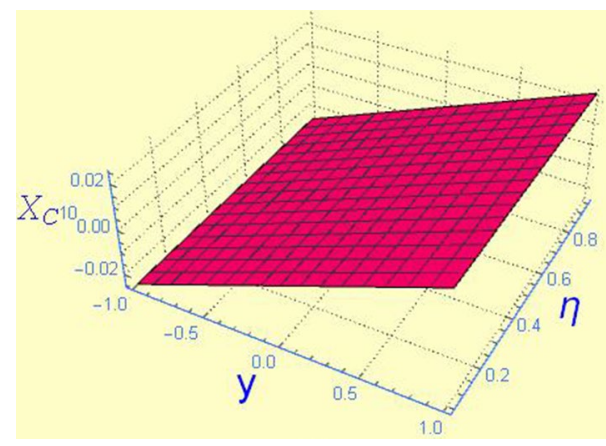

Fig. 14 Thermodynamic force $X_{C}$ versus $y$ and $\eta$ for $K n=0.75$, $M=0.5$

we must have $\varphi \equiv \varphi_{0}$. That gives a good qualitative agreement in the manner of the distribution function in [40]. The gas particles move far from the bottom plate due to the centrifugal force columnar to the base plate directed to the top one. It also runs from the top plate toward the bottom one due to the heat transfer from the hotter plate to the cold one. So, the gas concentration is growing in the middle between the two plates, especially in the strong centrifugal field, as shown in Fig. 4a, b. In Fig. 4c, the gas concentration is symmetric about the center axis between the two plates. In Fig. 4d, the concentration has its minimum value near the top plate (the hotter one). It increases nonlinearly with $\boldsymbol{\eta}$ near the top plate, while it decreases nonlinearly with $\eta$ near the bottom plate.

At the small Knudsen number values (such as Kn 0.01 to 0.1 relatively dense gases), the effect of centrifugal forces on the particles is magnified. Thus, the concentration decreases nonlinearly when the gas was moving from the bottom plate to the upper plate. When we had a value of the relatively large Knudsen number (such as $K n 1$ to 2.5 for a rarified low-density gas), we find that the centrifugal forces decrease their effect on the variation of gas concentration. Thus, the concentration decreases linearly when the gas was moving from the bottom plate to the upper plate, as shown in Fig. $4 \mathrm{e}, \mathrm{f}$.

In Figs. 4 and 5a-f, the temperature acts in an opposite scenario manner compared with the concentration manner. That is due to the uniform pressure as in the nondimensional form $P=n T=$ constant, which implies that $n$ is proportional to $1 / T$, so it is a regular manner in this case that their style acts against each other.

In Fig. 5a, temperature is growing gradually nonlinearly as the gas moves away from the bottom plate to the top one at a small value of $(M=0.1$ to 0.5$)$. It decreases nonlinearly with $y$ until it reaches the center point between the two plates, then it is reversing its direction, and it increases nonlinearly toward the top plate at high values of $(M=0.5$ to 1$)$. Obviously, in Fig. 5b, the temperature acts in the same manner with the high amounts of ( $M=0.5$ to 1 ). In Fig. $5 c$, the temperature manner is symmetric about the center axis between the two plates. In Fig. $5 \mathrm{~d}$, the temperature has its maximum value near the top plate (the hotter one). It decreases nonlinearly with $\eta$ near the top plate, while it increases nonlinearly with $\eta$ near the bottom plate. In this manner, a temperature gradient will set up across the gas. But, the temperature gradient will create a density gradient in the opposite direction. From Figs. $4 \mathrm{e}$, f and $5 \mathrm{e}$, $\mathrm{f}$, the temperature and the concentration bear up the equilibrium value with increasing the Knudsen number. We found the same manner did the entropy. It increases until it reaches its maximum value (the equilibrium value), as shown in Fig. 6 , which sheds light upon the harmonization between the three surfaces of entropy $S$, temperature $T$, and concentration $n$.

As we illustrated before, in Figs. 4 and 5a-f, the concentration acts oppositely with the temperature. Accordingly, the thermodynamic force (caused by the gradient of temperature $X_{T}$ ) will have an inverse direction with the thermodynamic force (caused by the slope of the density $\left.X_{n}\right)$, as shown in Figs. 12 and 13. Again, this is due to the uniform pressure. Let us illustrate that behavior in a mathematical proof as follows:

$$
\begin{aligned}
P= & n T=\text { constant } \Rightarrow \ln (P)=\ln (n)+\ln (T)=C_{1}: \therefore \frac{\partial \ln (P)}{\partial y}=\frac{\partial \ln (n)}{\partial y}-\frac{\partial \ln (T)}{\partial y}=0 \\
\Rightarrow & \frac{1}{n} \frac{\partial n}{\partial y}=-\frac{1}{T} \frac{\partial T}{\partial y} \text { But } X_{n}=\frac{H}{n} \frac{\partial n}{\partial y} \& X_{T}=\frac{H}{T} \frac{\partial T}{\partial y} \Rightarrow X_{n}=-X_{T} .
\end{aligned}
$$


It is evident from the temperature and concentration surfaces in Figs. 4 and 5a, $b$ that the non-equilibrium manner is a maximum in the middle distance between the two plates, so we find that the entropy had minimum value in the same zone. Figure 6 shows that the entropy $S$ is an increasing function with the temperature ratio parameter until it reaches its maximum value. That is due to that the more the difference between the two plate temperatures decreases, the more the gas reaches the equilibrium state, and then the more the gas entropy increases. Figure 7 shows that the entropy production $\sigma$ is a decreasing function with the temperature ratio parameter until it reached its minimum value when $\eta$ tended to one. So, the entropy production $\sigma$ managed its equilibrium value (minimum) when $\eta$ tended to its equilibrium value $(\eta=1)$. The same behavior is done by the entropy $S$ when it tended to its equilibrium value (maximum) when $\eta$ tended to its equilibrium value $(\eta=1)$. That manner illustrated the harmony between the irreversible non-equilibrium thermodynamic parameters. Manifestly, Fig. 7 shows that the entropy production $\sigma$ is a nonnegative function. This is confirmed with the second law of thermodynamics and the Boltzmann $\mathrm{H}$-theorem. Evidently, Figs. 6 and 7 illustrate that the system goes to equilibrium, which proved with Le Chatelier's principle.

We found that the manner of the different participations of the change in internal energies can be illustrated as follows: The internal energy changes caused by the variations in the temperature $\mathrm{d} U_{S}$ and caused by the differences in the concentration $\mathrm{d}_{V}$ have opposite signs. They have the same direction as the thermodynamic forces $X_{T}$ and $X_{n}$, which are formed by the gradient of temperature and density, respectively. They decrease the magnitude toward the equilibrium state $(\eta=1)$, as shown in Figs. 8, 9, 12, and 13. The internal energy change $\mathrm{d} U_{V}$ caused by the variation in the density has a positive sign. It takes the same direction as the thermodynamic force $X_{n}$, which is caused by the gradient of density.

The numerical ratios between the different participations of the internal energy variation based on the derivatives of the extensive parameters calculated via the Gibbs equation illustrated in Figs. 8, 9, and 10 are ordered in magnitude as (those calculations are performed for the argon gas only but in different cases):

In the case of $K n=0.75, \eta=0.5$, and $M$ varying from 0.1 to 1 , which represents the weak-moderated intensity of the external centrifugal force, we obtained: $\mathrm{d} U_{s}: \mathrm{d} U_{V}: \mathrm{d} U_{C} \sim 1: 1.0510^{-1}: 1.0510^{-1}$, where the $\mathrm{d} U_{S}$ participation is the dominant one.

In the case of $K n=0.75, \eta=0.5$, and $M$ varying from 1 to 1.5 , which represents the vigorous relativity intensity of the external centrifugal force, we obtained: $\mathrm{d} U_{s}: \mathrm{d} U_{V}: \mathrm{d} U_{C} \sim 1: 4.8210^{-2}: 7.24110^{-2}$, where the $\mathrm{d} U_{S}$ participation is the dominant one.

In the case of $K n=0.75, M=0.5$, and $\eta$ varying from 0.1 to 0.9 , which represents the variable intensity of the temperature differences between the two parallel rigid plane plates in several cases, we obtained: $\mathrm{d} U_{s}: \mathrm{d} U_{V}: \mathrm{d} U_{C} \sim 1: 4.7510^{-2}: 7.510^{-3}$, where the $\mathrm{d} U_{S}$ participation is the dominant one.

In the case of $\eta=0.5, M=0.1$, and $K n$ varying from 0.1 to 1 in the transition regime where the neutral gas is a rarefied, we obtained: $\mathrm{d} U_{s}: \mathrm{d} U_{V}: \mathrm{d} U_{C} \sim 1: 810^{-2}: 3.510^{-4}$, where the $\mathrm{d}_{S}$ participation is the dominant one.

In the case of $\eta=0.5, M=0.1$, and $K n$ varying from 0.01 to 0.1 in the slip regime where the neutral gas is a weak rarefied gas, we obtained: $\mathrm{d} U_{s}: \mathrm{d} U_{V}: \mathrm{d} U_{C} \sim 1: 8.3310^{-2}: 3.510^{-3}$, where the $\mathrm{d} U_{S}$ participation is the dominant one.

The change in internal energy $d U_{S}, d U_{V}$, and $d U$ decreases the magnitude which tends to zero, as shown in Figs. 8, 9, and 11, and they go toward the equilibrium state, which gives an agreement with Le Chatelier's principle. The same manner and the same deal with Le Chatelier's principle hold for the thermodynamic forces $X_{T}$ and $X_{n}$, as shown in Figs. 12 and 13. Figure 14 illustrates that the thermodynamic force (caused by the centrifugal force $X_{C}$ ) did not depend on $\eta$ but depended only on $y$. That can easily be deduced from its formula. It is dependent only on $y$ and the centrifugal Mach number $M$. It increases nonlinearly with $M$ and linearly with $y$.

For the nine kinetic coefficients, we found some critical notices on their manner. The negative sign on some dynamic factors, related to cross-effects, means that there exists a heat flux in the opposite direction to the flux caused by the imposed thermodynamic force (gradient). For example, we had a negative sign in front of $L_{T n}=L_{12}$ and $L_{n T}=L_{21}$, which means that there exists flow generated by the temperature gradient, from the bottom temperature value to the higher temperature value, which is known as a thermal diffusion (or Soret effect), that gives a qualitative agreement in a general manner with [9, 21-23, 39].

The Onsager-Casimir reciprocal relations are satisfied in some kinetic coefficients and are not satisfied with all dynamic factors due to the external centrifugal force. Obviously, $L_{T T}=L_{n n}$ in all cases in the whole range of radial Mach number, for the full range of the Knudsen number and all values of temperature ratio parameter. $L_{C C} \geq 0$. In all cases, the kinetic inequality $L_{11} L_{22}-\frac{1}{4}\left(L_{21}-L_{12}\right)^{2} \cong 0$ as it is fluctuating in the order between $\pm 10^{-14}$ to $\pm 10^{-17}$ that is an acceptable error for our model. 


\section{Conclusions}

The effectiveness of an external centrifugal force such as Pomraning's model and the heat transfer, on the performance of neutral gas, confined between two parallel horizontal rigid fixed plane plates, is presented in the steady state. For this approach, an exact analytical solution of the BGK (Bhatnagar-Gross-Krook) kinetic model is introduced. In a frame co-moving with the fluid, the system of four nonlinear unsteady differential equations is solved. The moment method is utilized. The temperature and concentration are calculated. The difference between the perturbed distribution functions and the equilibrium distribution function is investigated. The thermodynamic predictions are discussed. The internal energy change in the system is examined. The results are employed for laboratory argon neutral gas. Threedimensional and two-dimensional graphics are drawn to clarify their manner. We found qualitative agreements with previous related studies.

We concluded that:

- the decrement in $\varphi_{2}$ and the increment in $\varphi_{1}$ of the distribution functions $\varphi$ are due to the non-equilibrium state according to the effect of the external centrifugal force and the heat transfer between the two rigid plates. That is interpreted as the departure from the equilibrium state, where the gas particles having temperature $T_{1}$ and density $n_{1}$, near the bottom plate after affected by an external centrifugal force, are replaced by a countercurrent of gas particles having temperature $T_{2}$ and density $n_{2}$ near the outer plate after affecting by the heat transfer from the top plate (the hotter one). This manner agrees with Le Chatelier's principle.

- At the small Knudsen number values, as Knudsen number decreases, there will be more collective behaviors from particles than individual motions, and the force field acts more like a kinematic force on the fluid. Thus, the concentration decreases nonlinearly when the gas was moving from the bottom plate to the upper plate. When there is the value of the relatively large Knudsen number, we find that the centrifugal force decreases its effect on the variation of gas concentration, and thus, the concentration decreases linearly when the gas was moving from the bottom plate to the upper plate.

- When the gas is high rarefied, the $\mathrm{d} U_{S}$ participation in the total energy change is becoming the dominant one.

- Our model and all calculations were tested. We discovered that it is consistent with the second law of thermodynamics, Le Chatelier's principle, and the Boltzmann
$\mathrm{H}$-theorem. This examination was done with a wide range of the Knudsen numbers, the centrifugal Mach number, and the temperatures ratio parameter.

- Our model is satisfied the kinetic inequality $L_{11} L_{22}-\frac{1}{4}\left(L_{21}-L_{12}\right)^{2} \cong 0$ as it is fluctuating in the order between $\pm 10^{-14}$ to $\pm 10^{-17}$ that was a very acceptable error for our model. We illustrated that in the new conclusion.

- The negative sign on some dynamic factors related to cross-effects means that in these cases, there exists a heat flux in the opposite direction to the flux caused by the imposed thermodynamic force (gradient), which is consistent with the results obtained by $T_{i j}$ and Santos [41]. They showed that the external force induces heat transport along the force direction, even in the absence of gradients along that direction. Also, it agreed with the results obtained by Aoki et al. [42]. The same results are acquired by [43], where they conclude that the heat flux along the positive direction of external force acceleration has been enhanced. In other words, the external force will contribute the heat flux transport in the forcing direction. For example, we had a negative sign in front of $L_{T n}=L_{12}$ and $L_{n T}=L_{21}$, which means that there exists flow generated by the temperature gradient, from the bottom temperature value to the higher temperature value, which is known as a thermal diffusion (or Soret effect), that gives a qualitative agreement in a general manner with $[9,21-23,39]$.

- The Onsager-Casimir reciprocal relations are satisfied in some kinetic coefficients and are not satisfied with all dynamic factors due to the external centrifugal force. Obviously, $L_{\Pi}=L_{n n}$ in all cases in the whole range of radial Mach numbers, for the full range of the Knudsen number and all values of temperature ratio parameter. $L_{C C} \geq 0$ in all cases which is consistent with the second law of thermodynamics.

Acknowledgements The authors want to thank the reviewers, the editor, the assistant editors, and the whole family of the journal for their concern, for their valuable time, and for their useful comments that improved the paper value much more than before.

\section{Compliance with ethical standards}

Conflict of interest The authors declare that they have no competing interests.

\section{References}

1. Mikami H (1973) Thermally induced flow in gas centrifuge, (I). J Nucl Sci Technol 10(7):396-401 
2. Pomraning CG (1963) General electric technical information series; Rept., No. APED-4374, 63 APE 22

3. De Socio LM, Marino L (2000) Simulation and modelling of flows between rotating cylinders. Math Models Methods Appl Sci 10(1):73-83

4. Johnson EA (1983) Shear flow in the presence of 'strong rotation'. I. Exact free-molecular solution. J Phys D Appl Phys 16:1201. https://doi.org/10.1088/0022-3727/16/7/011

5. Johnson EA, Stopford PJ (1983) Shear flow in the presence of strong rotation: II. Approximation for continuum-plus-rarefied flow. J Phys D Appl Phys 16:1207-1215

6. Wahid TA (2017) The effect of Lorentz and centrifugal forces on gases and plasma: exact solutions, analytical methods, and real geometry. LAP LAMBERT Academic Publishing, ISBN-13: 978-6202055048

7. Sharipov F, Kalempa D (2008) Oscillatory Couette flow at arbitrary oscillation frequency over the whole range of the Knudsen number. Microfluid Nanofluid 4(5):363-374

8. Abourabia AM, Wahid TZA (2010) The unsteady Boltzmann kinetic equation and non-equilibrium thermodynamics of an electron gas for the Rayleigh flow problem. Can J Phys 88:501511. https://doi.org/10.1139/P10-032

9. Abourabia AM, Wahid TZA (2011) Solution of the Krook kinetic equation model and non-equilibrium thermodynamics of a rarefied gas affected by a non-linear thermal radiation field. $J$ Non-Equilibrium Thermodyn 36:75-98

10. Abourabia AM, Wahid TZA, J Non-Equilib Thermodyn, 37(1): 1-25. ISSN (Online) 1437-4358, ISSN (Print) 0340-0204. https:// doi.org/10.1515/jnetdy.2011.022

11. Wahid TAZ (2014) Kinetic irreversible thermodynamic study of plasma and neutral gases. LAP LAMBERT Academic Publishing, Germany, ISBN-13: 978-3659622960

12. Cao B-Y, Sun J, Chen M, Guo Z-Y (2009) Molecular momentum transport at fluid-solid interfaces in MEMS/NEMS: a review. Int J Mol Sci 10:4638-4706. https://doi.org/10.3390/ijms10114638

13. Wahid TZA, J Non-Equilib Thermod, 37(2): 119-141. ISSN (Online) 1437-4358, ISSN (Print) 0340-0204. https://doi.org/10.1515/ jnetdy.2011.034

14. Kalempa D, Sharipov F, Silva JC (2019) Sound waves in gaseous mixtures induced by vibro-thermal excitation at arbitrary rarefaction and sound frequency. Vacuum 159:82-98

15. Kalempa D, Sharipov F (2014) Numerical modeling of thermoacoustic waves in a rarefied gas confined between coaxial cylinders. Vacuum 109:326-332

16. Sharipov F, Dias FC (2019) Temperature dependence of shock wave structure in helium and neon. Phys Fluids 31:037109

17. Wahid TAZ (2018) Kinetic thermodynamic study of thermal radiation field effect on gases. LAP LAMBERT Academic Publishing, Germany. ISBN-13: 978-613-9-92664-0

18. Kalempa D, Sharipov F (2016) Sound propagation through a binary mixture of rarefied gases at arbitrary sound frequency. Eur J Mech B/Fluids 57:50-63

19. Wahid TZA, Elagan SK (2012) Kinetic treatment for the exact solution of the unsteady Rayleigh flow problem of a rarefied homogeneous charged gas bounded by an oscillating plate. Can J Phys 90:987-998

20. Wahid TZA (2013) Mathematical problems in engineering, vol 2013, Article ID 503729, 13 p, 2013. https://doi. org/10.1155/2013/503729

21. Wahid TZA (2013) Exact solution of the unsteady Krook kinetic model and nonequilibrium thermodynamic study for a rarefied gas affected by a nonlinear thermal radiation field. Can J Phys 91(3):201-210

22. Abourabia AM, Wahid TZA (2012) Kinetic and thermodynamic treatments of a neutral binary gas mixture affected by a nonlinear thermal radiation field. Can J Phys 90:137-149
23. Wahid TZA (2015) Travelling wave solution of the unsteady BGK model for a rarefied gas affected by a thermal radiation field. Sohag J Math 2(2):75-87. https://doi.org/10.12785/sjm/020205

24. Wahid TZA (2018) Travelling waves solution of the unsteady flow problem of a collisional plasma bounded by a moving plate. Fluid Mech 4(1):27-37. https://doi.org/10.11648/j.fm.20180 401.14

25. Ballester JL, Alexeev I, Collados M et al (2018) Partially ionized plasmas in astrophysics. Space Sci Rev 214:58. https://doi. org/10.1007/s11214-018-0485-6

26. Bekezhanovaa VB, Goncharova ON (2018) Problems of evaporative convection (review). Fluid Dyn 53(Suppl. 1):S69-S102

27. Ho MT, Graur I (2015) Heat transfer through rarefied gas confined between two concentric spheres. Int J Heat Mass Transf 90:58-71

28. Jakobsen HA (2014) Elementary kinetic theory of gases. In: Chemical reactor modeling. Springer, Cham

29. Sharipov F (2016) Rarefied gas dynamics: fundamentals for research and practice. Wiley-VCH, London

30. Wahid TZA (2019) Kinetic and thermodynamic study of the thermal radiation effect on the gases. LAMBERT Academic Publishing, Germany., ISBN: ISBN 978-613-9-92664-0

31. Lees L (1965) Kinetic theory description of rarefied gas flow. J Soc Ind Appl Math 13:278-311

32. Wahid TZA (2014) Travelling waves solution of the unsteady problem of binary gas mixture affected by a nonlinear thermal radiation field. Am J Phys Appl 2(6):121-134. https://doi. org/10.11648/j.ajpa.20140206.13

33. Rieutord M (2015) Fluid dynamics. Springer, Cham. https://doi. org/10.1007/978-3-319-09351-2

34. Brouwers JJH (1978) On compressible flow in a gas centrifuge and its effect on the maximum separative power. Nucl Technol 39:311-322

35. Olander DR (1981) The theory of uranium enrichment by the gas centrifuge. Prog Nucl Energy 8(1):1-33

36. Wahid TZA (2017) Kinetic and thermodynamic treatments of unsteady gaseous plasma flows. LAMBERT Academic Publishing, Germany, ISBN: 978-613-9-90736-6

37. Abramowitz M, Stegun IA (eds) (1972) Handbook of mathematical functions with formulas, graphs, and mathematical tables, 9th printing. New York: Dover, pp 295 and 319

38. Jou D, Casas-Vázquez J, Lebon G (2010) Extended irreversible thermodynamics. Springer, Berlin

39. Naris S, Valougeorgis D, Kalempa D, Sharipov F (2004) Phys A 336(3-4):294-318

40. Cheremisin FG (1970) "Verification of the nature of the approximation of the Boltzmann integral by Krook's kinetic relaxation model" Moscow. Translated from Izvestiya Akademii Nauk SSSR, Mekhanika Zhidkosti i Gaza, (4), 3-7

41. Tij M, Santos A (1994) Perturbation analysis of a stationary nonequilibrium flow generated by an external force. J Stat Phys 76:1399-1414. https://doi.org/10.1007/BF02187068

42. Aoki K, Takata S, Nakanishi T (2002) Poiseuille-type flow of a rarefied gas between two parallel plates driven by a uniform external force. Phys RevE 65:026315

43. Xiao T, Xu K, Cai Q, Qian T (2018) An investigation of non-equilibrium heat transport in a gas system under external force field. Int J Heat Mass Transf 126:362-379

Publisher's Note Springer Nature remains neutral with regard to jurisdictional claims in published maps and institutional affiliations. 